\title{
Plasma Expansion Dynamics in Hydrogen Gas
}

\author{
Ghaneshwar Gautam ${ }^{1}$ (i) and Christian G. Parigger ${ }^{2, *}$ (i) \\ 1 Fort Peck Community College, 605 Indian Avenue, Poplar, MT 59255, USA; ggautam@fpcc.edu \\ 2 Department of Physics and Astronomy, University of Tennessee/University of Tennessee Space Institute, \\ 411 B.H. Goethert Parkway, Tullahoma, TN 37388, USA \\ * Correspondence: cparigge@tennessee.edu; Tel.: +1-931-841-5690
}

Received: 13 July 2018; Accepted: 10 August 2018; Published: 20 August 2018

\begin{abstract}
Micro-plasma is generated in ultra-high-pure hydrogen gas, which fills the inside of a cell at a pressure of $(1.08 \pm 0.033) \times 10^{5}$ Pa by using a Q-switched neodymium-doped yttrium-aluminum-garnet (Nd:YAG) laser device operated at a fundamental wavelength of $1064 \mathrm{~nm}$ and a pulse duration of $14 \mathrm{~ns}$. The micro-plasma emission spectra of the hydrogen Balmer alpha line, $\mathrm{H}_{\alpha}$, are recorded with a Czerny-Turner type spectrometer and an intensified charge-coupled device. The spectra are calibrated for wavelength and corrected for detector sensitivity. During the first few tens of nanoseconds after the initiation of optical breakdown, the significant Stark-broadened and Stark-shifted $\mathrm{H}_{\alpha}$ lines mark the well-above hypersonic outward expansion. The vertical diameters of the spectrally resolved plasma images are measured for the determination of expansion speeds, which were found to decrease from 100 to $10 \mathrm{~km} / \mathrm{s}$ for time delays of 10 to $35 \mathrm{~ns}$. For time delays of $0.5 \mu \mathrm{s}$ to $1 \mu \mathrm{s}$, the expansion speed of the plasma decreases to the speed of sound of $1.3 \mathrm{~km} / \mathrm{s}$ in the near ambient temperature and pressure of the hydrogen gas.
\end{abstract}

Keywords: laser-plasma interactions; plasma dynamics and flow; hypersonic flows; Emission Spectra

\section{Introduction}

In laser-induced plasma, the ambient gas can foster or diminish the plasma expansion. The pressure and type of gases also influences the post-breakdown phenomena. For example, at low pressures, the losses and uniformity of the plasma energy distribution increases [1]. Plasma size, propagation speed and emission properties are also related to the ambient gas into which the plasma expands. The physical cause of the high-speed expansion is the large pressure difference between the plasma and its surrounding environment.

This work investigates the expansion dynamics of plasma in hydrogen gas at a pressure that is slightly above $10^{5} \mathrm{~Pa}$. The study of the plasma expansion is applicable for astrophysical, engineering and scientific research as well as other various applications [2-5]. The astrophysical interests include the interpretation of white dwarf photosphere absorption spectra by simulating the conditions of these selected stars in laboratory settings [2-4] and by exploring laser-induced plasma [4] with time-resolved laser spectroscopy. The motivation behind this engineering and scientific research [5] extends to the measurement of phenomena that occur at hypersonic speeds to re-entry speeds that are in the Mach number range of $5-25$ or above.

During the plasma expansion, the spatial and temporal variations of density, temperature and pressure are observed. Investigations of these spatial and temporal profiles allow us to infer the expansion speeds. Plasma at elevated temperatures and pressures expands at a high velocity and causes a shock wave that propagates into the outward direction. As the incident laser beam is focused at a point above the breakdown threshold irradiance, the breakdown occurs at a location before the pulse reaches its focal point [6]. 
The interaction between the laser beam and the material is a complex process, which depends on many characteristics, such as laser parameters or target materials. Various factors affect this interaction, including the properties of the pulse, such as the pulse width, spatial and temporal fluctuation of the pulse, as well as the peak irradiance variations. For example, the effects of the pulse width on nascent laser-induced bubbles for underwater laser-induced breakdown spectroscopy (LIBS) show that a long pulse causes well-defined spectra with clear lines. In turn, a short pulse usually causes considerably asymmetric or deformed spectra. However, this effect is more significant for a solid target material than for a gaseous target material [7]. Moreover, the images of laser-induced breakdown plasma in air [8] nicely illustrate expansion dynamics.

The experimental results in air, which were obtained using a Nd:YAG laser at the wavelength of $532 \mathrm{~nm}$ and the pulse width of $6.5 \mathrm{~ns}$ [8], show two distinct regions with higher intensity towards the laser propagation direction for time delays of $25 \mathrm{~ns}$ to $10 \mu \mathrm{s}$. Schlieren images of laser-induced plasma generated in air at the standard ambient temperature and pressure also show plasma jet propagation towards the laser for time delays of 1-20 $\mu$ s [9]. However, the jet propagation direction depends on the type of gas, the gas pressure, the ratio of energy absorbed in the plasma and the threshold irradiance for the occurrence of optical breakdown [9].

\section{Results}

This section elaborates on the determination of the expansion speed of the micro-plasma, which was generated in the ultra-high-pure hydrogen gas inside a cell. Experimental results show high plasma expansion speeds that decreased from 100 to $10 \mathrm{~km} / \mathrm{s}$ at early time delays of $10 \mathrm{~ns}$ to $35 \mathrm{~ns}$.

Figure 1 displays the recorded $\mathrm{H}_{\alpha}$ plasma spectra at early time delays of $10 \mathrm{~ns}$ to $35 \mathrm{~ns}$, which were measured at 5-ns time intervals. The 2-dimensional spectra of slit-height versus wavelength are significantly Stark-broadened and Stark-shifted at early time delays. The measured intensity increases for successive time delays. However, the area and line-width of the spectral profiles decrease continuously, which implies decreasing electron density. Based on the recorded $\mathrm{H}_{\alpha}$ plasma spectra at early time delays as displayed in Figure 1, plasma expansion speeds were determined. The diameter of the plasma in the lateral or slit-height direction is measured as a function of time and hence, the plasma expansion-speed can be determined. For example, the red arrows on the spectra images at $10 \mathrm{~ns}$ and $15 \mathrm{~ns}$ in Figure 1 indicate the spatial plasma ranges used for the determination of the expansion speeds.

(a)

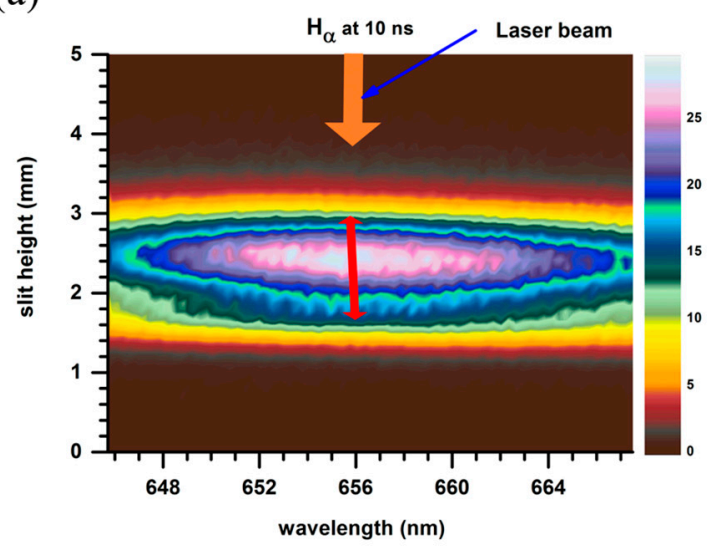

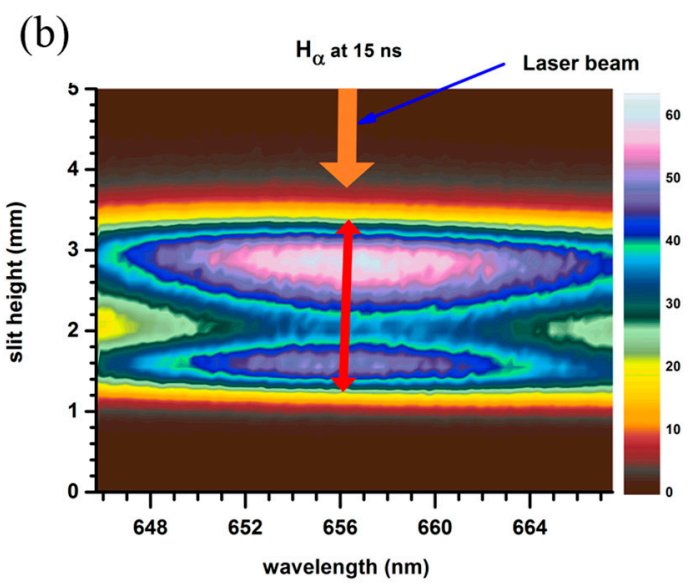

Figure 1. Cont. 
(c)

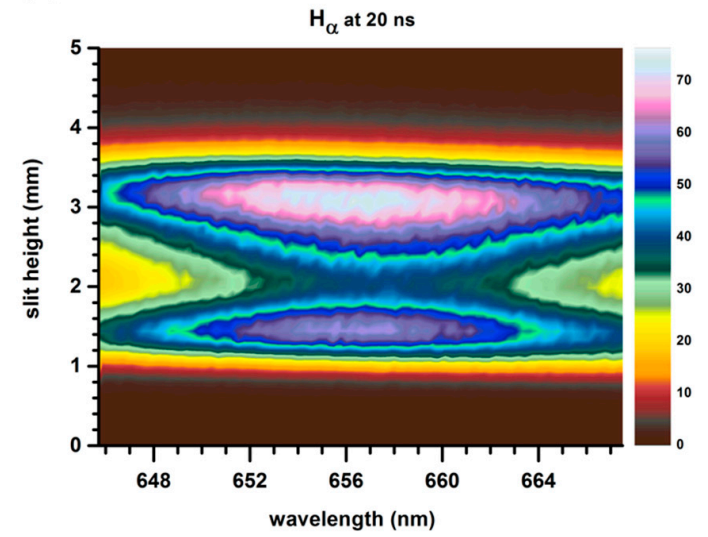

(e)

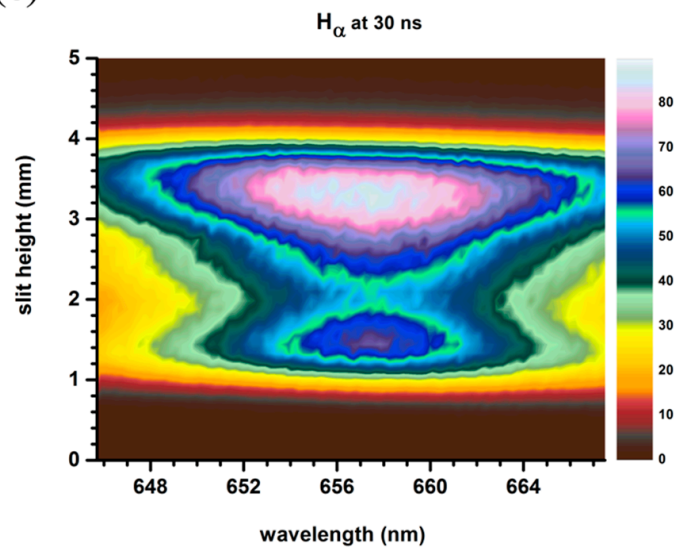

(d)

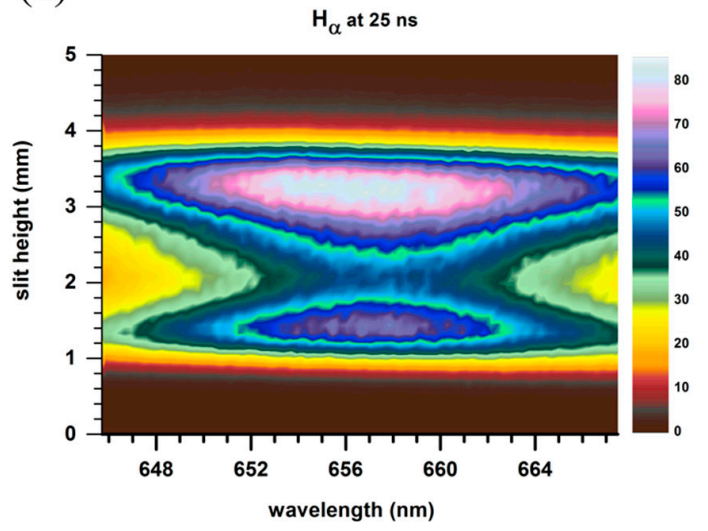

(f)

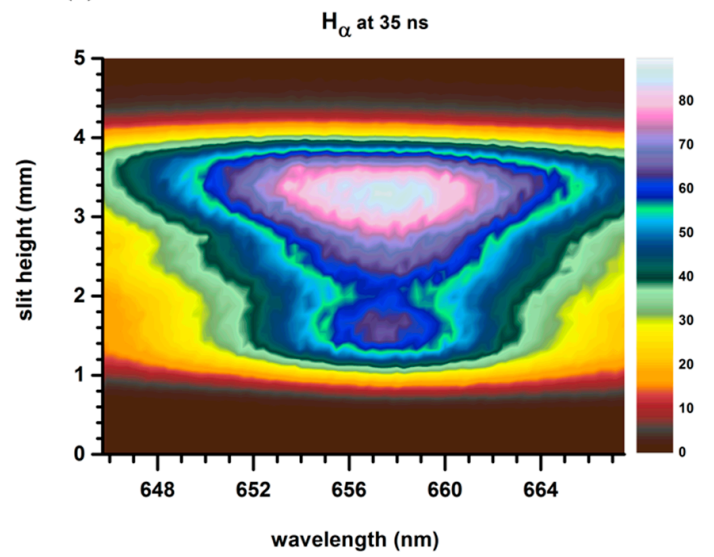

Figure 1. Hydrogen alpha plasma spectra images for selected time delays: (a) $10 \mathrm{~ns}$; (b) $15 \mathrm{~ns}$; (c) 20 ns; (d) $25 \mathrm{~ns}$; (e) $30 \mathrm{~ns}$; and (f) $35 \mathrm{~ns}$.

The recorded images of the laser-induced plasma with 14-ns pulses depict a higher intensity towards the laser at early time delays of $10 \mathrm{~ns}$ to $35 \mathrm{~ns}$. However, the opposite behavior is observed at later time delays, e.g., for time delays of $400 \mathrm{~ns}$. Figure 2 illustrates the experimental records for time delays of 400 and $900 \mathrm{~ns}$.

(a)

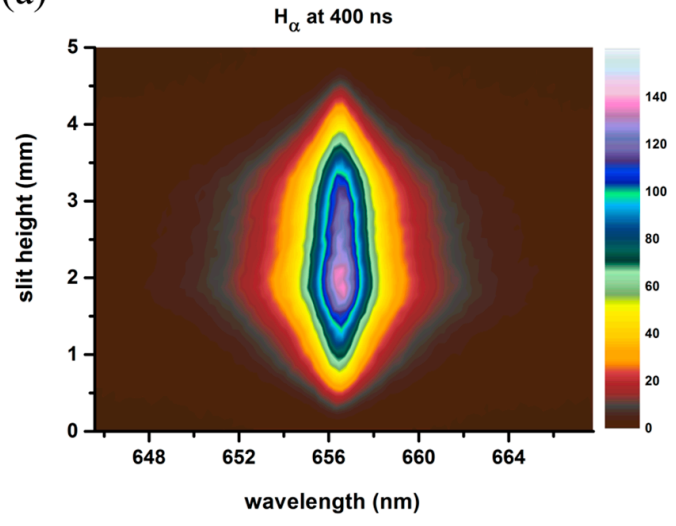

(b)

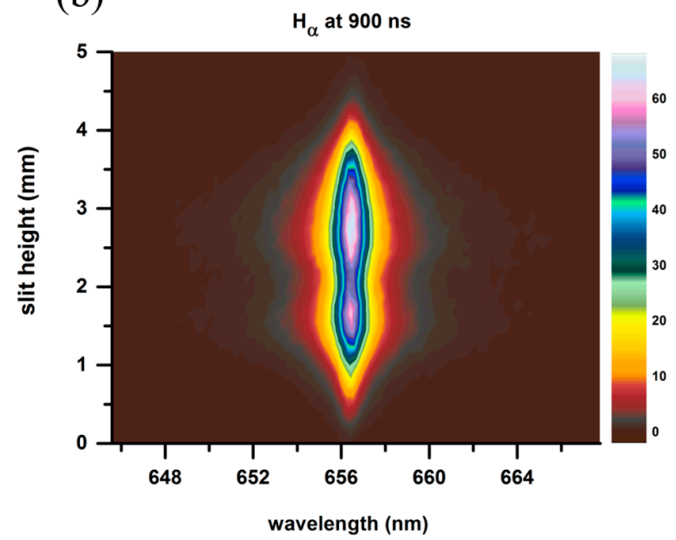

Figure 2. Hydrogen alpha plasma spectra images at time delays of (a) $400 \mathrm{~ns}$ and (b) $900 \mathrm{~ns}$. 
From the images in Figure 1, the diameter of the plasma in the lateral or slit-height direction is measured as a function of time and hence, plasma expansion speeds can be determined. Table 1 displays the measured diameters of the plasma and the corresponding speeds at various time delays.

Table 1. Plasma expansion speed at various time delays using lateral direction expansion from the contour plots exhibited in Figures 1 and 2.

\begin{tabular}{cccc}
\hline $\begin{array}{c}\text { Time } \\
(\mathbf{n s})\end{array}$ & $\begin{array}{c}\text { Diameter } \\
(\mathbf{m m})\end{array}$ & $\begin{array}{c}\text { Distance in } \mathbf{5} \mathbf{~ n s} \\
\mathbf{( m m )}\end{array}$ & $\begin{array}{c}\text { Speed } \\
\mathbf{( k m} / \mathbf{s})\end{array}$ \\
\hline 10 & 1.32 & -- & -- \\
15 & 2.03 & 0.71 & 142 \\
20 & 2.42 & 0.39 & 78 \\
25 & 2.71 & 0.29 & 58 \\
30 & 2.86 & 0.15 & 30 \\
35 & 2.92 & 0.06 & 12 \\
400 & 0.96 & -- & -- \\
900 & 1.81 & $0.85^{*}$ & 1.70 \\
\hline
\end{tabular}

${ }^{*}$ Distance is for $500 \mathrm{~ns}$.

The predicted plasma expansion speeds decrease from 100 to $10 \mathrm{~km} / \mathrm{s}$ for time delays of 15 to $35 \mathrm{~ns}$. The determined expansion speeds are well above the hypersonic speed (Mach number 5) or above the re-entry speeds (Mach number 25) at these time delays. Figure 3 illustrates the tabulated results.

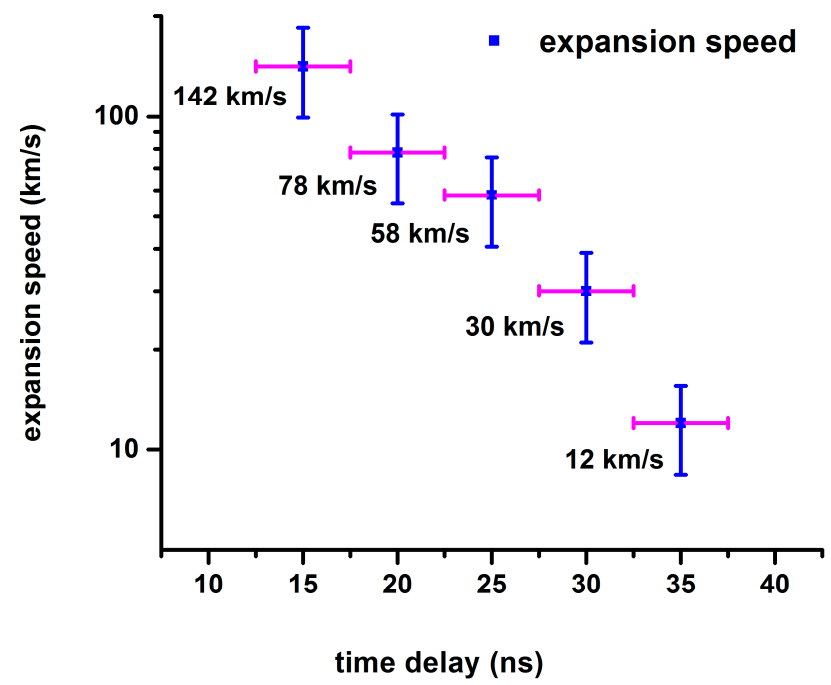

Figure 3. Plasma expansion speeds in log scale (see Table 1). The indicated time-delay error bars are due to the gate width of 5 ns.

The plasma expansion typically decreases to hypersonic and sonic speeds for larger time delays as indicated in Figure 3. The measured expansion speeds are consistent with the results from previous experimental studies [10,11]. However, computer simulations show a shock-wave expansion speed of $60 \mathrm{~km} / \mathrm{s}$ at a time delay of $20 \mathrm{~ns}$ in air at atmospheric pressure [12], thereby indicating that the determined speeds are consistent with other experimental results. It is important to note that the speed of sound in hydrogen gas is $1.3 \mathrm{~km} / \mathrm{s}$, which is 3.5 -fold higher than in the ambient pressure and temperature of the air.

For longer time delays of about $1 \mu \mathrm{s}$, the images in Figure 2 are utilized for the determination of the plasma expansion speed. The determined speed is $1.7 \pm 0.5 \mathrm{~km} / \mathrm{s}$ at $900 \mathrm{~ns}$. The estimated error bars for these speeds are $\pm 30 \%$. These results agree well with those of the recent hydrogen experiments [13]. 


\section{Materials and Methods}

During the experiment, a cell is filled with ultra-high-pure hydrogen gas at a pressure of $(1.08 \pm 0.033) \times 10^{5} \mathrm{~Pa}$. To study time-resolved and space-resolved emission spectroscopy, a Q-switched Nd:YAG laser device (Quanta Ray DCR-2A (10), Spectra-Physics, Santa Clara, CA, USA [14]) is used at its fundamental wavelength of $1064 \mathrm{~nm}$ with $10-\mathrm{Hz}$ repetition rate and full-width-half-maximum pulses of $14 \mathrm{~ns}$. The measured energy per pulse is $120 \mathrm{~mJ}$. The laser beam was passed through a dichroic beam splitter to remove the residual 532-nm component. A silicon photodiode detector was used to record a portion of the laser radiation that is reflected off of the beam splitter at the exit of the laser source. The photodiode is connected to an oscilloscope to monitor the optical pulse. Three mirrors (NB1-K13; Thorlabs, Newton, NJ, USA [4]) are used to align the beam to be parallel to the spectrometer slit. Figure 4 illustrates the experimental schematic.

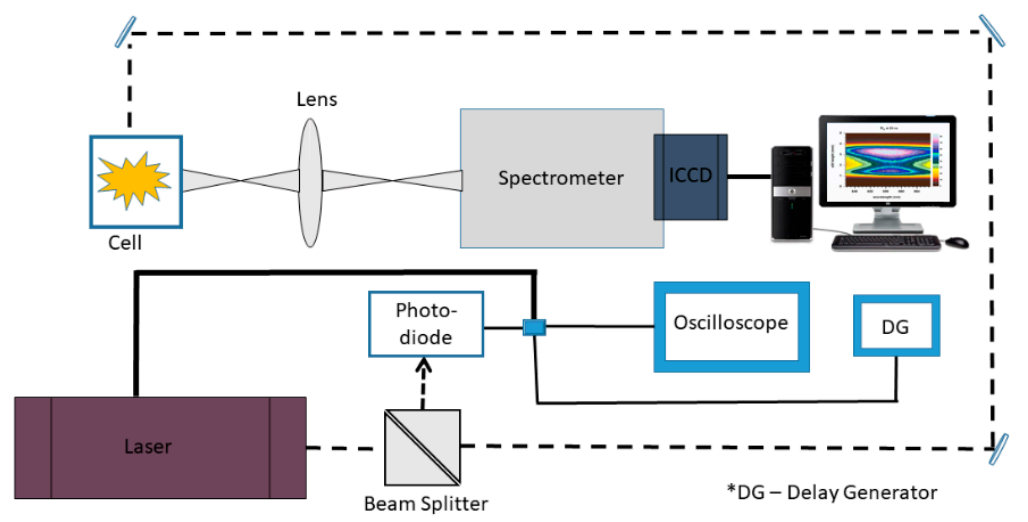

Figure 4. Experimental schematic for time-resolved laser spectroscopy.

A holographic grating of 1200 grooves $/ \mathrm{mm}$ is selected to disperse the radiation from the plasma. For the recording of temporally and spatially resolved plasma emission spectra images along the slit height, the following instrumentation is employed: Czerny-Turner type spectrometer (0.64-m HR640; Jobin-Yvon, Longjumeau, FR [4]) and intensified charge-coupled device (ICCD) (Andor technology model iStar DH334T-25U-03, South Windsor, CT, USA [4]). The spectral resolution was $0.11 \mathrm{~nm}$. The data were recorded with a 5-ns gate width and an average of 100 consecutive laser-plasma events were accumulated. For later time delays of $400 \mathrm{~ns}$ and $900 \mathrm{~ns}, 50$ consecutive laser-plasma events were accumulated using a 20-ns gate width. The recorded spectra are wavelength calibrated and corrected for detector sensitivity. Matlab ${ }^{\circledR}$ (Mathworks, Nattick, MA, USA [4]) scripts are utilized for wavelength calibration and sensitivity correction, while Origin Software (OriginLab, Northampton, MA, USA [4]) is employed for the graphical display of the experimental data.

\section{Conclusions}

The laser-induced plasma expands at a well-above hypersonic speed depending upon the ambient conditions and time delays. The spectra recorded during the evolution of the plasma are significantly Stark-broadened and Stark-shifted. Therefore, a larger percentage error occurs for the predicted speeds for earlier time delays. For longer time delays, the plasma expansion speeds decrease considerably and thus, larger differences occur for the speed measurement due to the increased temporal interval. To improve the graphically inferred expansion speeds, Abel inversion methods can be applied so that radial information will be extracted from the recorded line-of-sight measurements [14]. The predicted expansion speeds may be useful for the National Aeronautics and Space Administration (NASA) hypersonic technology (HT) project. Details of the focal volume irradiance distribution [15] for the study of laser-induced optical breakdown may augment future experimental studies. 
Author Contributions: C.G.P. and G.G. designed and performed the experiments. G.G. analyzed the result and wrote the paper with suggestions from C.G.P.

Funding: This work is in part supported by the Center for Laser Applications, a State of Tennessee supported Center of Excellence, at The University of Tennessee Space Institute.

Conflicts of Interest: The authors declare no conflict of interest.

\section{References}

1. Anabitarte, F.; Cobo, A.; Lopez-Higuera, J.M. Laser-induced breakdown spectroscopy: Fundamentals, applications, and challenges. Int. Sch. Res. Notices Spectrosc. 2012, 2012, 285240. [CrossRef]

2. Falcon, R.E.; Rochau, G.A.; Bailey, J.E.; Ellis, J.L.; Carlson, A.L.; Gomez, T.A.; Montgomery, M.H.; Winget, D.E.; Chen, E.Y.; Gomez, M.R.; et al. An experimental platform for creating white dwarf photospheres in the laboratory. High Energy Density Phys. 2013, 9, 82-90. [CrossRef]

3. Falcon, R.E.; Rochau, G.A.; Bailey, J.E.; Gomez, T.A.; Montgomery, M.H.; Winget, D.E.; Nagayama, T. Laboratory measurements of white dwarf photospheric spectral lines: $\mathrm{H}_{\beta}$. Astrophys. J. 2015, 806, 214. [CrossRef]

4. Parigger, C.G.; Drake, K.A.; Helstern, C.M.; Gautam, G. Laboratory hydrogen-beta emission spectroscopy for analysis of astrophysical white dwarf spectra. Atoms 2018, 6, 36. [CrossRef]

5. Engeln, R.; Mazouffre, S.; Vankan, P.; Bakker, I.; Schram, D.C. Plasma expansion: fundamentals and applications. Plasma Sources Sci. Technol. 2002, 11, A100. [CrossRef]

6. Chen, Y.L.; Lewis, J.W.L.; Parigger, C.G. Spatial and temporal profiles of pulsed laser-induced air plasma emissions. J. Quant. Spectrosc. Radiat. Transf. 2000, 67, 91-103. [CrossRef]

7. Sakka, T.; Tamura, A.; Matsumoto, A.; Fukami, K.; Nishi, N.; Thornton, B. Effects of pulse width on nascent width on nascent laser-induced bubbles for underwater laser-induced breakdown spectroscopy. Spectrochim. Acta Part B At. Spectrosc. 2014, 97, 94-98. [CrossRef]

8. Glumac, N.; Elliott, G.; Boguszko, M. Temporal and spatial evolution of a laser spark in air. Am. Inst. Aeronaut. Astronaut. J. 2005, 34, 1984-1994. [CrossRef]

9. Brieschenk, S.; O’Byrne, S.; Kleine, H. Visualization of jet development in laser-induced plasmas. Opt. Lett. 2013, 38, 664-666. [CrossRef] [PubMed]

10. Parigger, C.G.; Plemmons, D.H.; Lewis, J.W.L. Spatially and temporally resolved electron number density measurements in a decaying laser-induced plasma using hydrogen-alpha line profiles. Appl. Opt. 1995, 34, 3325-3330. [CrossRef] [PubMed]

11. Parigger, C.G. Atomic and molecular emissions in laser-induced breakdown spectroscopy. Spectrochim. Acta Part B At. Spectrosc. 2013, 79, 4-16. [CrossRef]

12. Sobral, H.; Villagrán-Muniz, M.; Navarro-González, R.; Raga, A.C. Temporal evolution of the shock wave and hot core air in laser induced plasma. Appl. Phys. Lett. 2000, 77, 3158-3160. [CrossRef]

13. Parigger, C.G.; Surmick, D.M.; Gautam, G. Self-absorption characteristics of measured laser-induced plasma line shapes. J. Phys. Conf. Ser. 2017, 810, 012012. [CrossRef]

14. Parigger, C.G.; Gautam, G.; Surmick, D.M. Radial electron density measurements in laser-induced plasma from Abel inverted hydrogen Balmer beta line profiles. Int. Rev. At. Mol. Phys. 2015, 6, 43-55.

15. Parigger, C.G. Laser-induced breakdown in gases: Experiments and simulation. In Laser-Induced Breakdown Spectroscopy; Miziolek, A.W., Palleschi, V., Schechter, I., Eds.; Cambridge University Press: Cambridge, UK, 2006; ISBN 978-0521071000.

(C) 2018 by the authors. Licensee MDPI, Basel, Switzerland. This article is an open access article distributed under the terms and conditions of the Creative Commons Attribution (CC BY) license (http:// creativecommons.org/licenses/by/4.0/). 